\title{
SOME INEQUALITIES AMONG GENERAIIZED DIVERGENCE MEASURES
}

\author{
I.J.TANEJA, L.PARDO AND M.L.MENÉNDEZ
}

\begin{abstract}
Burbea and Rao [3,4] and Sgarro [11] established an inequality between two famous divergence measures i.e., Jeffreys [6] invariant function ( $J$-divergence) and Sibson's [13] information radius ( $R$-divergence). Taneja [14,16] generalized both these divergences ( $J$ and $R$ ) having two scalar parameters. In this paper, we have extended the inequality between the $R$ and $J$-divergences for two parametric cases. For one parametric generalizations of $R$ and $J$-divergences, the generalized inequalities are improved.
\end{abstract}

\section{Introduction}

Let

$$
\Delta_{n}=\left\{p=\left(p_{1}, p_{2}, \ldots, p_{n}\right), p_{i}>0, \sum_{i=1}^{n} p_{i}=1\right\}, \quad n \geq 2
$$

be the set of all complete finite discrete probability distributions.

Sibson [13] introduced a measure of information for two probability distributions, called information radius given by

$$
R(P \| Q)=\sum_{i=1}^{n}\left[\frac{p_{i} \operatorname{Ln} p_{i}+q_{i} \operatorname{Ln} q_{i}}{2}-\left[\frac{p_{i}+q_{i}}{2}\right] \operatorname{Ln}\left[\frac{p_{i}+q_{i}}{2}\right]\right]
$$

for all $P, Q \in \triangle_{n}$.

The measure (1) arises due to the concavity property of Shannon's entropy. Sometimes, it is called $[3,4]$ the Jensen difference divergence measure. By simple calculations, we can write

$$
R(P \| Q)=\frac{1}{2}\left[D\left(P \| \frac{P+Q}{2}\right)+D\left(Q \| \frac{P+Q}{2}\right)\right]
$$

for all $P, Q \in \triangle_{n}$, where $D(P \| U)$ is the well-known Kullback-Leibler's [7] directed divergence, given by

$$
D(P \| U)=\sum_{i=1}^{n} p_{i} \operatorname{Ln} \frac{p_{i}}{u_{i}}
$$


for all $P, U \in \triangle_{n}$ with $U=\frac{(P+Q)}{2}$. A symmetric version of (3) known as $J$-divergence (ref. Jeffreys [6]; Kullback and Leibler [7]) is given by

$$
J(P \| Q)=D(P \| Q)+D(Q \| P) .
$$

for all $P, Q \in \triangle_{n}$.

Burbea and Rao [3,4] and Sgarro [11] established an inequality between the measures (1) and (4) given by

for all $P, Q \in \triangle_{n}$.

$$
J(P \| Q) \geq 4 R(P \| Q)
$$

Recently, Taneja [16] presented one and two parametric generalizations of the measure (2). The one and two parametric generalizations of the measure (4) are already given before by Taneja [14]. Some parametric generalizations of the measures (1) and (4) are also studied by Burbea [1,2] and Burbea and Rao [3,4]. In the following two subsections we have specified some of these generalizations having one and two scalar parameters written in unified expressions.

\subsection{Unified $(r, s)$-Jensen Difference Divergence Measures}

For all $P, Q \in \triangle_{n}$, let us consider the following divergence measures:

defined by

$$
{ }^{t} R_{r}^{s}(P \| Q) \quad(r>0, t=1 \text { and } 2,)
$$

$$
\begin{aligned}
& { }^{1} R_{r}^{s}(P \| Q)=[2(s-1)]^{-1}\left\{\left[\sum_{i=1}^{n} p_{i}^{r}\left[\frac{p_{i}+q_{i}}{2}\right]^{1-r}\right]^{\frac{s-1}{r-1}}+\left[\sum_{i=1}^{n} q_{i}^{r}\left[\frac{p_{i}+q_{i}}{2}\right]^{1-r}\right]^{\frac{s-1}{r-1}}-2\right\}, \\
& { }^{2} R_{r}^{s}(P \| Q)=(s-1)^{-1}\left\{\left[\sum_{i=1}^{n}\left[\frac{p_{i}^{r}+q_{i}^{r}}{2}\right]\left[\frac{p_{i}+q_{i}}{2}\right]^{1-r}\right]^{\frac{s-1}{r-1}}-1\right\},
\end{aligned}
$$

when $r \neq 1, s \neq 1, r>0$,

with the boundary cases continuously extended by L'Hopital Rule:

$$
\begin{aligned}
{ }^{1} R_{1}^{s}(P \| Q)= & {[2(s-1)]^{-1}\left\{\exp _{e}\left[(s-1) \sum_{i=1}^{n} p_{i} \operatorname{Ln}\left[\frac{2 p_{i}}{p_{i}+q_{i}}\right]\right]\right.} \\
& \left.+\exp _{e}\left[(s-1) \sum_{i=1}^{n} p_{i} \operatorname{Ln}\left[\frac{2 p_{i}}{p_{i}+q_{i}}\right]\right]-2\right\}, \quad(s \neq 1) \\
{ }^{2} R_{1}^{s}(P \| Q)= & (s-1)^{-1}\left\{\exp _{e}[(s-1) R(P \| Q)]-1\right\}, \quad(s \neq 1) \\
{ }^{1} R_{r}^{1}(P \| Q)= & {[2(r-1)]^{-1} \operatorname{Ln}\left\{\left[\sum_{i=1}^{n} p_{i}^{r}\left[\frac{p_{i}+q_{i}}{2}\right]^{1-r}\right]\left[\sum_{i=1}^{n} q_{i}^{r}\left[\frac{p_{i}+q_{i}}{2}\right]^{1-r}\right]\right\}, } \\
& (r \neq 1, r>0)
\end{aligned}
$$


and

$$
{ }^{2} R_{r}^{1}(P \| Q)=(r-1)^{-1} \operatorname{Ln}\left\{\sum_{i=1}^{n}\left[\frac{p_{i}^{r}+q_{i}^{r}}{2}\right]\left[\frac{p_{i}+q_{i}}{2}\right]^{1-r}\right\},(r \neq 1, r>0) .
$$

We call them the unified $(r, s)$-Jensen difference divergence measures. In particular, when $r=s$, we have

$$
\begin{aligned}
{ }^{1} R_{s}^{s}(P \| Q) & ={ }^{2} R_{s}^{s}(P \| Q)=R_{s}^{s}(P \| Q) \\
& =(s-1)^{-1}\left\{\sum_{i=1}^{n}\left[\frac{p_{i}^{s}+q_{i}^{s}}{2}\right]\left[\frac{p_{i}+q_{i}}{2}\right]^{1-s}-1\right\}, \quad s \neq 1, s>0
\end{aligned}
$$

It can easily be checked (ref. Taneja [16]) that the measures ${ }^{t} R_{r}^{s}(P \| Q)(t=1$ and 2) are nonnegative for all $r>0$ and any $s$. For more properties of the measures given by (6) such as convexity, Schur-convexity, monotonicity with respect to parameters, data processing inequalities etc. refer to Menéndez et al. [8].

All the measures appearing in (6) are due to Taneja [16]. While, the measure (7) was studied before by Taneja [17]. For applications of the measures (6) to comparison of experiments, Fisher measure of information and statistical pattern recognition refer to [8] [9] [19].

\subsection{Umified $(r, s)$-J-Divergence Measures}

For all $P, Q \in \triangle_{n}$, let us consider the following divergence measures:

$$
{ }^{t} J_{r}^{s}(P \| Q) \quad(r>0, t=1 \text { and } 2),
$$

defined by

$$
\begin{gathered}
{ }^{1} J_{r}^{s}(P \| Q)=(s-1)^{-1}\left\{\left[\sum_{i=1}^{n} p_{i}^{r} q_{i}^{1-r}\right]^{\frac{s-1}{r-1}}+\left[\sum_{i=1}^{n} p_{i}^{1-r} q_{i}^{r}\right]^{\frac{s-1}{r-1}}-2\right\}, \\
{ }^{2} J_{r}^{s}(P \| Q)=2(s-1)^{-1}\left\{\left[\sum_{i=1}^{n}\left[\frac{p_{i}^{r} q_{i}^{1-r}+p_{i}^{1-r} q_{i}^{r}}{2}\right]\right]^{\frac{s-1}{r-1}}-1\right\}, \\
\text { when } r \neq 1, s \neq 1, r>0,
\end{gathered}
$$

with boundary cases:

$$
\begin{aligned}
{ }^{1} J_{1}^{s}(P \| Q)= & (s-1)^{-1}\left\{\exp _{e}\left[(s-1) \sum_{i=1}^{n} p_{i} \operatorname{Ln} \frac{p_{i}}{q_{i}}\right]\right. \\
& \left.+\exp _{e}\left[(s-1) \sum_{i=1}^{n} q_{i} \operatorname{Ln} \frac{q_{i}}{p_{i}}\right]-2\right\}, s \neq 1 \\
{ }^{2} J_{1}^{s}(P \| Q)= & 2(s-1)^{-1}\left\{\exp _{e}\left[\left(\frac{s-1}{2}\right) J(P \| Q)\right]-1\right\}, s \neq 1 \\
{ }^{1} J_{r}^{1}(P \| Q)= & (r-1)^{-1} \operatorname{Ln}\left\{\left[\sum_{i=1}^{n} p_{i}^{r} q_{i}^{1-r}\right]\left[\sum_{i=1}^{n} q_{i}^{r} p_{i}^{1-r}\right]\right\}, r \neq 1, r>0
\end{aligned}
$$


and

$$
{ }^{2} J_{r}^{1}(P \| Q)=2(r-1)^{-1} \operatorname{Ln}\left\{\sum_{i=1}^{n}\left[\frac{p_{i}^{r} q_{i}^{1-r}+p_{i}^{1-r} q_{i}^{r}}{2}\right]\right\}, \quad r \neq 1, r>0
$$
have

We call them the unified $(r, s)$-J-divergence measures. In particular, when $r=s$, we

$$
\begin{aligned}
{ }^{1} J_{s}^{s}(P \| Q) & ={ }^{2} J_{s}^{s}(P \| Q)=J_{s}^{s}(P \| Q) \\
& =(s-1)^{-1}\left\{\sum_{i=1}^{n}\left[p_{i}^{s} q_{i}^{1-s}+p_{i}^{1-s} q_{i}^{s}\right]-2\right\}, s \neq 1, s>0
\end{aligned}
$$

It can easily be checked (ref. Taneja [16]) that the measures ${ }^{t} J_{r}^{s}(P \| Q)(t=1$ and 2) are nonnegative for all $r>0$ and any $s$. For more properties of the measures given by (8) such as convexity, Schur-convexity, monotonicity with respect to parameters, generalized data processing inequalities etc. refer to Taneja et al. [18]. For applications of these measures (8) to comparison of experiments, Fisher measure of information and statistical pattern recognition refer to [15] [16].

Most of the measures appearing in the unified expression (8) are due to Taneja [16], except the measures ${ }^{1} J_{r}^{1}(P \| Q)$ and $J_{s}^{s}(P \| Q)$. The measure ${ }^{1} J_{r}^{1}(P \| Q)$ is due to Burbea [2] and the measure $J_{s}^{s}(P \| Q)$ is due to Burbea and Rao [3,4] and Rathie and Sheng [10].

In this paper our aim is to generalize the inequality (5) for the measures given by (6) and (8). Some mixed inequalities are also given.

\section{Inequalities for generalized divergence measures.}

In this section we generalize the inequality (5) for the measures (6) and (8). For one parametric generalizations of $R$ and $J$, the general results are improved.

Theorem 1. For all $P, Q \in \triangle_{n}$, we have

$$
{ }^{t} J_{r}^{s}(P \| Q) \geq 2{ }^{t} R_{r}^{s}(P \| Q) \quad(t=1 \text { and } 2)
$$

for all $r>0$ and any $s$.

Proof.

Case $t=1$.

Let $P=\left(p_{1}, \ldots, p_{n}\right) \in \triangle_{n}$ and $Q=\left(q_{1}, \ldots, q_{n}\right) \in \triangle_{n}$ be two probability distributions. Then by using Jensen inequality, we can write

$$
\frac{p_{i}^{1-r}+q_{i}^{1-r}}{\dot{2}}\left\{\begin{array}{l}
\leq\left[\frac{p_{i}+q_{i}}{2}\right]^{1-r}, \quad 0<1-r \dot{<} 1 \\
\geq\left[\frac{p_{i}+q_{i}}{2}\right]^{1-r}, \quad 1-r>1 \text { or } 1-r<0
\end{array}\right.
$$


for all $i=1,2, \cdots, n$. Multiplying by $p_{i}^{r}$ and summing over all $i=1,2, \cdots, n$, we get

$$
\sum_{i=1}^{n} p_{i}^{r}\left[\frac{p_{i}^{1-r}+q_{i}^{1-r}}{2}\right] \begin{cases}\leq \sum_{i=1}^{n} p_{i}^{r}\left[\frac{p_{i}+q_{i}}{2}\right]^{1-r}, & 0<r<1 \\ \geq \sum_{i=1}^{n} p_{i}^{r}\left[\frac{p_{i}+q_{i}}{2}\right]^{1-r}, \quad r>1\end{cases}
$$

i.e.,

$$
\frac{1}{2}\left[1+\sum_{i=1}^{n} p_{i}^{r} q_{i}^{1-r}\right] \begin{cases}\leq \sum_{i=1}^{n} p_{i}^{r}\left[\frac{p_{i}+q_{i}}{2}\right]^{1-r}, & 0<r<1 \\ \geq \sum_{i=1}^{n} p_{i}^{r}\left[\frac{p_{i}+q_{i}}{2}\right]^{1-r}, & r>1\end{cases}
$$

Similarly, we can get

$$
\frac{1}{2}\left[1+\sum_{i=1}^{n} q_{i}^{r} p_{i}^{1-r}\right] \begin{cases}\leq \sum_{i=1}^{n} q_{i}^{r}\left[\frac{p_{i}+q_{i}}{2}\right]^{1-r}, & 0<r<1 \\ \geq \sum_{i=1}^{n} q_{i}^{r}\left[\frac{p_{i}+q_{i}}{2}\right]^{1-r}, & r>1\end{cases}
$$

It is easy to check that

$$
\sum_{i=1}^{n} p_{i}^{r} q_{i}^{1-r} \begin{cases}\leq 1, & 0<r<1 \\ \geq 1, & r>1\end{cases}
$$

Also, it can easily be checked that if $A \leq 1$, then $A \leq \frac{A+1}{2}$ and if $A \geq 1$, then $A \geq \frac{A+1}{2}$. This gives,

$$
\sum_{i=1}^{n} p_{i}^{r} q_{i}^{1-r} \begin{cases}\leq \frac{1}{2}\left[1+\sum_{i=1}^{n} p_{i}^{r} q_{i}^{1-r}\right], & 0<r<1 \\ \geq \frac{1}{2}\left[1+\sum_{i=1}^{n} p_{i}^{r} q_{i}^{1-r}\right], & r>1\end{cases}
$$

From (11) and (14) we have

$$
\sum_{i=1}^{n} p_{i}^{r} q_{i}^{1-r} \begin{cases}\leq \sum_{i=1}^{n} p_{i}^{r}\left[\frac{p_{i}+q_{i}}{2}\right]^{1-r}, & 0<r<1 \\ \geq \sum_{i=1}^{n} p_{i}^{r}\left[\frac{p_{i}+q_{i}}{2}\right]^{1-r}, & r>1\end{cases}
$$

Similarly, from (12) we can get

$$
\sum_{i=1}^{n} q_{i}^{r} p_{i}^{1-r} \begin{cases}\leq \sum_{i=1}^{n} q_{i}^{r}\left[\frac{p_{i}+q_{i}}{2}\right]^{1-r}, & 0<r<1 \\ \geq \sum_{i=1}^{n} q_{i}^{r}\left[\frac{p_{i}+q_{i}}{2}\right]^{1-r}, & r>1\end{cases}
$$


Raising both sides of (15) and (16) by $\frac{s-1}{r-1}(r \neq 1, s \neq 1)$, and adding we get

$$
\begin{aligned}
& {\left[\sum_{i=1}^{n} p_{i}^{r} q_{i}^{1-r}\right]^{\frac{s-1}{r-1}}+\left[\sum_{i=1}^{n} q_{i}^{r} p_{i}^{1-r}\right]^{\frac{s-1}{r-1}}} \\
& \begin{cases}\leq\left[\sum_{i=1}^{n} p_{i}^{r}\left[\frac{p_{i}+q_{i}}{2}\right]^{1-r}\right]^{\frac{s-1}{r-1}}+\left[\sum_{i=1}^{n} q_{i}^{r}\left[\frac{p_{i}+q_{i}}{2}\right]^{1-r}\right]^{\frac{s-1}{r-1}}: & (0<r \neq 1, s-1<0) \\
\geq\left[\sum_{i=1}^{n} p_{i}^{r}\left[\frac{p_{i}+q_{i}}{2}\right]^{1-r}\right]^{\frac{s-1}{r-1}}+\left[\sum_{i=1}^{n} q_{i}^{r}\left[\frac{p_{i}+q_{i}}{2}\right]^{1-r}\right]^{\frac{s-1}{r-1}} \cdot & (0<r \neq 1, s-1>0)\end{cases}
\end{aligned}
$$

Subtracting 2 on both sides of (17), multiplying by $(s-1)^{-1}(s \neq 1)$, and simplifying, we get

$$
{ }^{1} J_{r}^{s}(P \| Q) \geq 2{ }^{1} R_{r}^{s}(P \| Q)
$$

for all $r>0, s \neq 1, r \neq 1$.

Case $t=2$.

Adding (11) and (12), we get

$$
1+\frac{1}{2} \sum_{i=1}^{n}\left[p_{i}^{r} q_{i}^{1-r}+q_{i}^{r} p_{i}^{1-r}\right] \begin{cases}\leq \sum_{i=1}^{n}\left(p_{i}^{r}+q_{i}^{r}\right)\left[\frac{p_{i}+q_{i}}{2}\right]^{1-r} & 0<r<1 \\ \geq \sum_{i=1}^{n}\left(p_{i}^{r}+q_{i}^{r}\right)\left[\frac{p_{i}+q_{i}}{2}\right]^{1-r} & r>1\end{cases}
$$

Similar to (13), we can check that

$$
\frac{1}{2} \sum_{i=1}^{n}\left[p_{i}^{r} q_{i}^{1-r}+q_{i}^{r} p_{i}^{1-r}\right] \begin{cases}\leq 1, & 0<r<1 \\ \geq 1, & r>1\end{cases}
$$
Again using the fact that, if $A \leq 1$, then $A \leq \frac{A+1}{2}$ and if $A \geq 1$, then $A \geq \frac{A+1}{2}$, from
(21), we get

$$
\frac{1}{2} \sum_{i=1}^{n}\left[p_{i}^{r} q_{i}^{1-r}+p_{i}^{1-r} q_{i}\right] \begin{cases}\leq\left\{1+\frac{1}{2} \sum_{i=1}^{n}\left[p_{i}^{r} q_{i}^{1-r}+q_{i}^{r} p_{i}^{1-r}\right]\right\}, & 0<r<1 \\ \geq\left\{1+\frac{1}{2} \sum_{i=1}^{n}\left[p_{i}^{r} q_{i}^{1-r}+q_{i}^{r} p_{i}^{1-r}\right]\right\} & r>1\end{cases}
$$

From (20) and (22), we get

$$
\frac{1}{2} \sum_{i=1}^{n}\left[p_{i}^{r} q_{i}^{1-r}+q_{i}^{r} p_{i}^{1-r}\right] \begin{cases}\leq \sum_{i=1}^{n}\left[\frac{p_{i}^{r}+q_{i}^{r}}{2}\right]\left[\frac{p_{i}+q_{i}}{2}\right]^{1-r}, & 0<r<1 \\ \geq \sum_{i=1}^{n}\left[\frac{p_{i}^{r}+q_{i}^{r}}{2}\right]\left[\frac{p_{i}+q_{i}}{2}\right]^{1-r}, & r>1\end{cases}
$$


Raising both sides of (23) by $\frac{s-1}{r-1}(s \neq 1, r \neq 1)$, we get

$$
\begin{aligned}
& {\left[\sum_{i=1}^{n}\left[\frac{p_{i}^{r} q_{i}^{1-r}+p_{i}^{1-r} q_{i}^{r}}{2}\right]\right]^{\frac{s-1}{r-1}}} \\
& \begin{cases}\leq\left[\sum_{i=1}^{n}\left[\frac{p_{i}^{r}+q_{i}^{r}}{2}\right]\left[\frac{p_{i}+q_{i}}{2}\right]^{1-r}\right]^{\frac{s-1}{r-1}} & (0<r \neq 1, s-1<0) \\
\geq\left[\sum_{i=1}^{n}\left[\frac{p_{i}^{r}+q_{i}^{r}}{2}\right]\left[\frac{p_{i}+q_{i}}{2}\right]^{1-r}\right]^{\frac{s-1}{r-1}} & (0<r \neq 1, s-1<0)\end{cases}
\end{aligned}
$$

Subtracting 1 on both sides of (24), multiplying by $(s-1)^{-1}(s \neq 1)$ and simplifying we get

$$
{ }^{2} J_{r}^{s}(P \| Q) \geq 2{ }^{2} R_{r}^{s}(P \| Q)
$$

for $r>0, s \neq 1, r \neq 1$.

By continuous extensions (25) and (18) are valid for all $r>0$ and any $s$.

In particular, when $r=s=1$, then from theorem 1 , we get

$$
J(P \| Q) \geq 2 R(P \| Q)
$$

It is quite obvious that the inequality (5) is much better than the inequality (27). In the following theorem, we shall improve the results of theorem 1 in some particular cases i.e., when $r=s$ and $s=1, r \neq 1$, and shall obtain the inequalities similar to (5).

Theorem 2. For all $P, Q \in \triangle_{n}$, we have

(i) $J_{s}^{s}(P \| Q) \geq 4 R_{s}^{s}(P \| Q), s \neq 1, s>0$

(ii) ${ }^{1} J_{r}^{1}(P \| Q) \geq 4{ }^{1} R_{r}^{1}(P \| Q), \quad 0<r<1$

(iii) ${ }^{2} J_{r}^{s}(P \| Q) \geq 4^{2} R_{r}^{1}(P \| Q), \quad 0<r<1$

Proof. (i) Replacing $r$ by $s$ in (20), we have

$$
1+\frac{1}{2} \sum_{i=1}^{n}\left[p_{i}^{s} q_{i}^{1-s}+q_{i}^{s} p_{i}^{1-s}\right]\left\{\begin{array}{lc}
\leq \sum_{i=1}^{n}\left(p_{i}^{s}+q_{i}^{s}\right)\left[\frac{p_{i}+q_{i}}{2}\right]^{1-s}, & 0<s<1 \\
\geq \sum_{i=1}^{n}\left(p_{i}^{s}+q_{i}^{s}\right)\left[\frac{p_{i}+q_{i}}{2}\right]^{1-s}, & s>1
\end{array}\right.
$$
i.e.,

$$
\sum_{i=1}^{n}\left[p_{i}^{s} q_{i}^{1-s}+q_{i}^{s} p_{i}^{1-s}\right]-1 \begin{cases}\leq 2\left[\sum_{i=1}^{n}\left[\frac{p_{i}^{s}+q_{i}^{s}}{2}\right]\left[\frac{p_{i}+q_{i}}{2}\right]^{1-s}-1\right], & 0<s<1 \\ \geq 2\left[\sum_{i=1}^{n}\left[\frac{p_{i}^{s}+q_{i}^{s}}{2}\right]\left[\frac{p_{i}+q_{i}}{2}\right]^{1-s}-1\right], & s>1\end{cases}
$$

Multipliying both sides of $(28)$ by $(s-1)^{-1}(s \neq 1)$ and simplifying we get

$$
\begin{array}{ll}
\frac{J_{s}^{s}(P \| Q)}{2} \geq 2 R_{s}^{s}(P \| Q), & s \neq 1, s>0 \\
J_{s}^{s}(P \| Q) \geq 4 R_{s}^{s}(P \| Q), & s \neq 1, s>0
\end{array}
$$


This completes the proof of part (i).

(ii) We know that

$$
\left(A^{\frac{1}{2}}-1\right)^{2} \geq 0 \quad \text { for all } A \geq 0
$$

this gives

$$
A-2 A^{\frac{1}{3}}+1 \geq 0
$$

i.e.,

$$
A+i \geq 2 A^{\frac{1}{2}}
$$

Let

$$
A=\sum_{i=1}^{n} p_{i}^{r} q_{i}^{1-r}, \quad r>0
$$

then from (29), we have

$$
2\left[\sum_{i=1}^{n} p_{i}^{r} q_{i}^{1-r}\right]^{\frac{1}{2}} \leq 1+\sum_{i=1}^{n} p_{i}^{r} q_{i}^{1-r}, \quad r>0
$$

Similarly, we can write

$$
2\left[\sum_{i=1}^{n} q_{i}^{r} p_{i}^{1-r}\right]^{\frac{1}{2}} \leq 1+\sum_{i=1}^{n} q_{i}^{r} p_{i}^{1-r}, \quad r>0
$$

From (11) and (30), we have

$$
\left[\sum_{i=1}^{n} p_{i}^{r} q_{i}^{1-r}\right]^{\frac{1}{2}} \leq \sum_{i=1}^{n} p_{i}^{r}\left[\frac{p_{i}+q_{i}}{2}\right]^{1-r}, \quad 0<r<1
$$

Again, from (12) and (31), we have

$$
\left[\sum_{i=1}^{n} q_{i}^{r} p_{i}^{1-r}\right]^{\frac{1}{2}} \leq \sum_{i=1}^{n} q_{i}^{r}\left[\frac{p_{i}+q_{i}}{2}\right]^{1-r}, \quad 0<r<1
$$

Taking $\log ($.$) on both sides of (32) and (33) and adding, we get$

$$
\begin{aligned}
& \frac{1}{2} \operatorname{Ln}\left\{\left[\sum_{i=1}^{n} p_{i}^{r} q_{i}^{1-r}\right]\left[\sum_{i=1}^{n} q_{i}^{r} p_{i}^{1-r}\right]\right\} \\
\leq & \operatorname{Ln}\left\{\left[\sum_{i=1}^{n} p_{i}^{r}\left[\frac{p_{i}+q_{i}}{2}\right]^{1-r}\right]\left[\sum_{i=1}^{n} q_{i}^{r}\left[\frac{p_{i}+q_{i}}{2}\right]^{1-r}\right]\right\}, \quad 0<r<1
\end{aligned}
$$

Multiplying both sides of $(34)$ by $(r-1)^{-1}(r \neq 1)$, and simplifying we get

$$
\frac{1}{2}{ }^{1} J_{r}^{1}(P \| Q) \geq 2{ }^{1} R_{r}^{1}(P \| Q), 0<r<1
$$


i.e.,

$$
{ }^{1} J_{r}^{1}(P \| Q) \geq{ }^{1} R_{r}^{1}(P \| Q), 0<r<1
$$

This completes the proof of part (ii).

(iii) In (29), let us take

$$
A=\frac{1}{2}\left[\sum_{i=1}^{n}\left[p_{i}^{r} q_{i}^{1-r}+q_{i}^{r} \cdot p_{i}^{1-r}\right], \quad r>0\right.
$$

then from (12), we have

$$
2\left[\frac{1}{2} \sum_{i=1}^{n}\left[p_{i}^{r} q_{i}^{1-r}+q_{i}^{r} p_{i}^{1-r}\right]\right]^{\frac{1}{2}} \leq 1+\frac{1}{2} \sum_{i=1}^{n}\left[p_{i}^{r} q_{i}^{1-r}+q_{i}^{r} p_{i}^{1-r}\right], \quad r>0
$$

from (20) and (35); we have

$$
\left[\frac{1}{2} \sum_{i=1}^{n}\left[p_{i}^{r} q_{i}^{1-r}+q_{i}^{r} p_{i}^{1-r}\right]\right]^{\frac{1}{2}} \leq \sum_{i=1}^{n}\left[\frac{p_{i}^{r}+q_{i}^{r}}{2}\right]\left[\frac{p_{i}+q_{i}}{2}\right]^{1-r}, \quad 0<r<1,
$$

Taking $\log ($.$) on both sides of (36)$ and multiplying by $(r-1)^{-1}(r \neq 1)$, we get

$$
{ }^{2} J_{r}^{1}(P \| Q) \geq 4{ }^{2} R_{r}^{1}(P \| Q), \quad 0<r<1
$$

This completes the proof of part (iii). Hence, completes the theorem.

Theorem 3. The following mixed inequalities hold:

(a) For $s \geq r$, we have

(i) ${ }^{1} J_{r}^{s}(P \| Q) \geq{ }^{2} J_{r}^{s}(P \| Q) \geq 2{ }^{2} R_{r}^{s}(P \| Q)$,

(ii) ${ }^{1} J_{r}^{s}(P \| Q) \geq 2{ }^{1} R_{r}^{s}(P \| Q) \geq 2{ }^{2} R_{r}^{s}(P \| Q)$,

(b) For $s \leq r$, we have

(i) ${ }^{2} J_{r}^{s}(P \| Q) \geq{ }^{1} J_{r}^{s}(P \| Q) \geq{ }^{2} R_{r}^{s}(P \| Q)$,

(ii) ${ }^{2} J_{r}^{s}(P \| Q) \geq 2{ }^{2} R_{r}^{s}(P \| Q) \geq 2{ }^{1} R_{r}^{s}(P \| Q)$,

(c) For $0<r<1$, we have

(i) ${ }^{1} J_{r}^{1}(P \| Q) \geq{ }^{2} J_{r}^{1}(P \| Q) \geq 4^{2} R_{r}^{1}(P \| Q)$,

(ii) ${ }^{1} J_{r}^{1}(P \| Q) \geq 4^{1} J_{r}^{1}(P \| Q) \geq 4^{2} R_{r}^{1}(P \| Q)$.

Proof. We known that (ref. Taneja [16])

$$
{ }^{1} R_{r}^{s}(P \| Q) \begin{cases}\leq{ }^{2} R_{r}^{s}(P \| Q), & s \leq r \\ \geq{ }^{2} R_{r}^{s}(P \| Q), & s \geq r\end{cases}
$$

and

$$
{ }^{1} J_{r}^{s}(P \| Q) \begin{cases}\leq{ }^{2} J_{r}^{s}(P \| Q), & s \leq r \\ \geq{ }^{2} J_{r}^{s}(P \| Q), & s \geq r\end{cases}
$$

for all $r>0$ and any $s$. 
In view of the theorems 1 and 2 , and the inequalities (37) and (38), the proof follows immediately.

\section{Acknowledgement}

One of the author (I.J. Taneja) is thankful to the Universidad Complutense de Madrid, Departamento de Estadistica e I.O., Madrid, for providing facilities and financial support. The authors also thanks to referee for valuable comments on an earlier version of the paper.

\section{References}

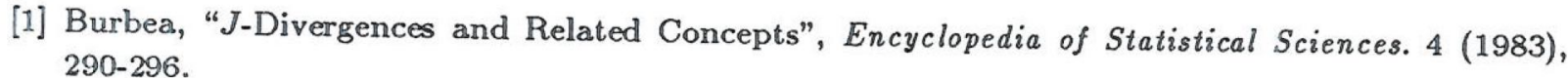

[2] Burbea, J., "The Convexity with Respect to Gaussian Distribution of Divergences of Order $\alpha$ ", Utilitas Mathematica. 26 (1984), 171-192.

[3] Burbea, J. and C. R. Rao, "On the convexity of some divergence measures based on entropy functions", IEEE Trans. on Information Theory. IT-28 (1982), 489-495.

[4] Burbea, J. and C. R. Rao, "Entropy Differential Metric, Distance and Divergence Measures in Probability Spaces: A Unified Approach", J. Multi. Analy. 12 (1982), 575-596.

[5] Capocelli, R. M. and I. J. Taneja, "Divergence Measures and Error Bounds", Proc. IEEE Interant. Conf. Syst., Man \& Cybern., Halifax, Canada, October 9-12 (1984), 43-46.

[6] H. Jeffreys, "An Invariant form for the Prior Probability in Estimation Problems". Proc. Royal Soc., Ser A. 186 (1946), 453-561.

[7] Kullback, S. and A. Leibler, "On the information and sufficiency". Ann. Math. Statist., 22 (1951), 79-86.

[8] Menendez, M. L., I. J. Taneja and L. Pardo, "On Unified $(r, s)$-Jensen Difference Divergence Measures", Communicated.

[9] Pardo, L., D. Morales, and I. J. Taneja, "Generalized Jensen Difference Divergence Mesures and Fisher Measure of Information", Communicated.

[10] Rathie, P. N. and L. T. Sheng, "The J-Divergence of Order $\alpha$ ", J. Comb. Inform. B Syst. Sci., 6 (1981), 197-205. [11] Sgarro, A., Informational divergence and the dissimilarity of probability distributions, Estratto di
Calcolo, XVIII (1981), 293-302.

[12] Shannon, C. E., "A Mathematical theory of Communication", Bell. Syst. Tech. J., 27 (1948),
379-423.

[13] Sibson, R., "Information Radius", Z. Wars. und verw Geb., 14 (1969), 149-160. [14] Taneja, I. J., "On a Characterization of J-Divergence and its Generalizations", J. Comb. Inform
\& Syst. Sci., 8 (1983), 206-212.

[15] Taneja, I. J., "Statistical aspects of divergence measures", J. Statist. Planning \& Inference, 16 (1987), 136-145.

[16] Taneja, I. J., "On Generalized Information Mesures and their Applications", Ad. Electronics and Electron Physics, 76 (1989), 327-413. [17] Taneja, I. J., "Bounds on the Probability of Error in Terms of Information Radius", Information
Sciences, Vol. 46. [18] Taneja, I. J., L. Pardo and M. L. Menendez, "On unified ( $r, s)$-J-divergence measures", Information
Sciences (under revision). 
[19] Taneja, I. J., L. Pardo and D. Morales, "Generalized Jensen Difference Divergence Measures and Comparison of Experiments", Communicated.

Departamento de Mat. Aplicada, E.T.S. de Arquitectura, U.P. de Madrid, 28040-Madrid, SPAIN (M.L. MENENDEZ).

Departmento de Estadistica e. I. O., Facultad de Matemáticas, Universidad Complutense de Madrid, 28040-Madrid, SPAIN (I.J. TANEJA and L.PARDO). 